\title{
Study of impulsive fractional differential equation under Robin boundary conditions by topological degree method
}

\author{
Atta Ullah', Kamal Shah', Thabet Abdeljawad ${ }^{2,3,4^{*}}$, Rahmat Ali Khan ${ }^{1}$ and Ibrahim Mahariq ${ }^{5}$
}

\author{
"Correspondence: \\ tabdeljawad@psu.edu.sa \\ ${ }^{2}$ Department of Mathematics and \\ General Sciences, Prince Sultan \\ University, Riyadh, Saudi Arabia \\ ${ }^{3}$ Department of Medical Research, \\ China Medical University, Taichung, \\ Taiwan \\ Full list of author information is \\ available at the end of the article
}

\begin{abstract}
This research work is dedicated to investigating a class of impulsive fractional order differential equations under the Robin boundary conditions via the application of topological degree theory (TDT). We establish some adequate results for the existence of at most one solution for the consider problem. Further, the whole analysis is illustrated by providing a pertinent example. We keep in mind that the conditions we develop by using TDT are much weaker than using ordinary fixed point theory. Hence TDT can be used as powerful tool for the theoretical analysis of many linear and nonlinear problems.
\end{abstract}

Keywords: Fractional differential equations; Impulsive problems; Robin boundary conditions; Topological degree theory

\section{Introduction}

In previous few decades, the area devoted to studying fractional calculus and derivatives and integrals of real or complex order has got proper attention. Fractional ordinary differential equations (FODEs) have numerous applications in the fields of physical science, biological sciences and engineering disciplines (for further details, see [1-7] and the references therein). The concerned area has the ability to model hereditary and memory process of many real world problems more comprehensively than classical derivatives and integral. Recently, some new differential operators of fractional order of nonsingular type have also attracted attention. Various researchers are working on it, it having also many applications in modeling real world problems. In this regard, some authors recently published very good work [8-10].

In real world problems, many phenomena face abrupt or sudden changes in their state of motion or rest. These abrupt changes are modeled by using impulsive differential equations. The area which addresses the aforementioned problems has been established very well in respect of ordinary derivatives and integrals. For the concerned investigations, researchers have used fixed point theory and nonlinear analysis tools. Further some results regarding numerical analysis corresponding to impulsive problems have also established $[11,12]$. On the other hand the investigation of impulsive problems under the concept of

(c) The Author(s) 2020. This article is licensed under a Creative Commons Attribution 4.0 International License, which permits use, sharing, adaptation, distribution and reproduction in any medium or format, as long as you give appropriate credit to the original author(s) and the source, provide a link to the Creative Commons licence, and indicate if changes were made. The images or other third party material in this article are included in the article's Creative Commons licence, unless indicated otherwise in a credit line to the material. If material is not included in the article's Creative Commons licence and your intended use is not permitted by statutory regulation or exceeds the permitted use, you will need to obtain permission directly from the copyright holder. To view a copy of this licence, visit http://creativecommons.org/licenses/by/4.0/. 
fractional calculus has also developed properly. The problem related to the moments of sudden changes like heartbeat, earthquake, temperature, shock, and so forth occurs in our daily life problems. All of these sudden changes are concerned with the impulsive differential equations, for these types of sudden changes the investigation of impulsive differential equations is an important tool for analysis. Large numbers of articles can be found in this regard [13-17].

An important aspect to be investigated of the physical problems is whether the problem physically exists or not. For the existence theory, fixed point theory has been utilized in the last many years. But one thing which restricted the application of fixed point theory is the requirement of strong conditions. Because when we deal with problems of integral or differential equations by fixed point theory, we first convert it to operator equations for which we search fixed points. To achieve this goal often we impose strong compact conditions on the concerned operator, which restricts the applicability of the area to a limited class of problems. Therefore it is necessary to search some more sophisticated methods and theories to relax the criteria. Therefore Mawhin [18] used TDT for the first time to deal with integral equations. Later on Isaia [19] used the same theory to investigate some integral equations. During 2012 Wang [20] investigated some classes of initial value problems of FODEs by using the TDT including the following problem of impulsive FODEs:

$$
\left\{\begin{array}{l}
D^{\gamma} \Psi(t)=h(t, \Psi(t)), \quad t \neq t_{i}, 0<\gamma \leq 1 \\
\delta \Psi\left(t_{i}\right)=I_{i}\left(\Psi_{i}\right), \\
\Psi(0)=\Psi_{0}
\end{array}\right.
$$

where $i=1,2, \ldots, l, h$ is a nonlinear function and $I_{i}: \mathbb{R} \rightarrow \mathbb{R}$ is a nonlinear map which is the size of the jump at $t_{i}$. As from present literature, the mentioned method has been used to investigate the usual initial and "boundary value problems" (BVPs) of FODEs (for details, see [21-23]).

On the other hand BVPs have many applications in engineering disciplines; see [24, 25]. The above-mentioned problems involve different kinds of boundary conditions like "Neumann boundary conditions and Dirichlet boundary conditions". When the aforesaid boundary conditions are mixed then for the problem the mixed boundary conditions are called Robin boundary conditions (RBCs) or impedance BVPs. The concerned problems have many applications in heat transfer phenomena and electromagnetic theory. Further problems under RBCs are increasingly used in solving Sturm-Liouville equations which are largely used in engineering disciplines (see [26, 27]). Keeping in mind the mentioned literature, we investigate the following nonlinear problem of impulsive FODEs under RBCs for $t \in[0, T]$ :

$$
\left\{\begin{array}{l}
D^{\gamma} \Psi(t)=h(t, \Psi(t)), \quad t \neq t_{i}, 1<\gamma \leq 2 \\
\delta \Psi\left(t_{i}\right)=I_{i}\left(\Psi\left(t_{j}\right)\right), \quad \delta \Psi^{\prime}\left(t_{i}\right)=J_{j}\left(\Psi\left(t_{i}\right)\right) \\
a \Psi(0)+b \Psi(T)=g_{1}(\Psi), \quad c \Psi^{\prime}(0)+d \Psi^{\prime}(T)=g_{2}(\Psi)
\end{array}\right.
$$

where $j=1,2, \ldots, l, 0<\gamma \leq 2$ and the nonlinear function $h$ is continuous and $I_{j}, J_{j}$ are nonlinear maps which determine the size of the jump at $t_{i}$, where $0<t_{1}<t_{0}<t_{2}<t_{3} \cdots<t_{l}$ and $I_{i}\left(\Psi\left(t_{i}\right)\right)=\Psi\left(t_{i}^{+}\right)-\Psi\left(t_{i}^{-}\right), J_{j}\left(\Psi^{\prime}\left(t_{i}\right)\right)=\Psi^{\prime}\left(t_{i}^{+}\right)-\Psi^{\prime}\left(t_{i}^{-}\right)$, the notations $\Psi\left(t_{i}^{+}\right), \Psi^{-}\left(t_{i}^{+}\right)$, and 
$\Psi\left(t_{i}^{-}\right), \Psi^{-}\left(t_{i}^{-}\right)$are right and left limits, respectively, and $D^{\gamma}$ represents the Caputo derivative of various orders where $1<\gamma \leq 2$. We use TDT to establish some adequate results that ensure the existence of a solution to the considered problem. A pertinent example is given to demonstrate the main contribution.

\section{Background materials}

Some basic notions and results $[1,2]$ which we need for our analysis are given as follows.

Proposition 2.1 The following statements hold:

(1) $\psi(H)=0$, iff $H$ is relatively compact;

(2) $\psi$ is of semi-norm, that is, $\psi(\lambda H)=|\lambda| \psi(H)$ and $\psi\left(H_{1}+H_{2}\right) \leq \psi\left(H_{1}\right)+\psi\left(H_{2}\right)$;

(3) $H_{1} \subset H_{2}$ implies $\psi\left(H_{1}\right) \leq \psi\left(H_{2}\right) \psi\left(H_{1} U H_{2}\right)=\max \left\{\psi\left(H_{1}\right), \psi\left(H_{2}\right)\right\}$;

(4) $\psi(\operatorname{conv} H)=\psi(H)$;

(5) $\psi\left(H^{-}\right)=\psi(H)$.

Definition 2.1 Let $\omega \subset Y$ and $F: \omega \rightarrow Y$ a continuous bounded map, we say that $F$ is $\psi$-Lipschitz if there exists $k \geq 0$ such that $\psi(F(H)) \leq k \psi(H)$, for all $H \subset \omega$. If $k<1$, then we say that $F$ is a strict $\psi$-contraction.

Proposition 2.2 If $F_{1}, F_{2}, F_{3}, F_{4}: \omega \rightarrow Y$ are $\kappa$-Lipschitz maps with the constants $m_{1}, m_{2}$, $m_{3}, m_{4}$, respectively, then $F_{1}, F_{2}, F_{3}, F_{4}: \omega \rightarrow Y$ are $\kappa$-Lipschitz with the constant $m_{1}+m_{2}+$ $m_{3}+m_{4}$.

Proposition 2.3 If $G: \omega \rightarrow Y$ is a compact mapping, then we call $G \psi$-Lipschitz with zero constant.

Proposition 2.4 If $G: \omega \rightarrow Y$ is Lipschitz with the constant $k$, then $G$ is $\psi$-Lipschitz with the same constant $k$.

Theorem 2.1 Let $F: Y \rightarrow Y$ be $\psi$-condensing and

$$
T=\{\Psi \in Y \text { : there exists } \lambda \in[0, T] \text { such that } \Psi=\lambda F \Psi\} .
$$

If $T$ is a bounded set in $Y$, so there exists $r>0$ such that $T \subset B_{r}(0)$, then

$$
D\left(I-\lambda F, B_{r}(0), 0\right)=1 \quad \text { for all } \lambda \in[0, T]
$$

Then $F$ has at least one fixed point and the set of fixed points of $F$ lies in $B_{r}(0)$.

Lemma 2.1 The FODE wih $\gamma>0$,

$$
D^{\gamma} \Psi(t)=0
$$

has a unique solution of the form $\Psi(t)=\sum_{n=0}^{m} c_{i} t^{m-1}$, and $c_{i}$ are real constants and $n=$ $[\gamma]+1$. 
Lemma 2.2 The next consequence holds for FODE with $\gamma>0$ :

$$
I^{\gamma} D^{\gamma} x(t)=\Psi(t)+\sum_{i=0}^{m-1} \mathrm{c}_{i} t^{i}
$$

for arbitrary constant $\mathrm{c}_{i} \in \mathbb{R}, i=0,1,2,3, \ldots, m-1, m=[\gamma]+1$.

The notation $Y=\left\{\Psi:[0, T] \rightarrow \mathbb{R}: \Psi \in C\left(I^{\prime}\right)\right\}$ is used for the space. Further, $(Y,\|\cdot\|)$ is the Banach space under the norm $\|\Psi\|=\sup _{t \in[0, T]}|\Psi(t)|$.

\section{The existence results for the considered problem}

Here we define some hypotheses for the existence and uniqueness theory for the problem under consideration and we name the space $P C(J, R)=\Upsilon$; is needed throughout this work.

$\left(A_{1}\right)$ Let there for $\Psi_{1}, \Psi_{2} \in \Upsilon$ exist constants $K_{g_{1}}, K_{g_{2}} \in[0,1)$ such that

$$
\left|g_{1}\left(\Psi_{1}\right)-g_{1}\left(\Psi_{2}\right)\right| \leq K_{g_{1}}\left|\Psi_{1}-\Psi_{2}\right|, \quad\left|g_{2}\left(\Psi_{1}\right)-g_{2}\left(\Psi_{2}\right)\right| \leq K_{g_{2}}\left|\Psi_{1}-\Psi_{2}\right| .
$$

$\left(A_{2}\right)$ Let us for $\Psi \in \Upsilon$ have some constants $C_{g_{1}}, M_{g_{1}}, C_{g_{2}}, M_{g_{2}} \in[0,1)$ such that

$$
\left|g_{1}(\Psi)\right| \leq C_{g_{1}}|\Psi|+M_{g_{1}}, \quad\left|g_{2}(\Psi)\right| \leq C_{g_{2}}|\Psi|+M_{g_{2}} .
$$

$\left(A_{3}\right)$ Let us for $\Psi \in \Upsilon$ have some constants $C_{f}, M_{f} \in[0,1)$ such that

$$
|h(t, \Psi)| \leq C_{f}+|\Psi| M_{f}
$$

$\left(A_{4}\right)$ Let us for $\Psi \in \mathbb{R}$ have some constants $C_{1}, M_{1}, C_{2}, M_{2} \in[0,1)$ such that

$$
\left|I_{j}(\Psi)\right| \leq C_{1}|\Psi|+M_{1}, \quad\left|J_{j}(\Psi)\right| \leq C_{2}|\Psi|+M_{2}
$$

$\left(A_{5}\right)$ Let $I_{i}, J_{i}: \mathbb{R} \rightarrow \mathbb{R}$ and let us have some constants $K_{I}^{i}, K_{J}^{i} \in\left[0, \frac{1}{m}\right)$ such that

$$
\left|I_{i}\left(\Psi_{1}\right)-I_{i}\left(\Psi_{2}\right)\right| \leq K_{I}^{i}\left|\Psi_{1}-\Psi_{2}\right|, \quad\left|J_{i}\left(\Psi_{1}\right)-J_{i}\left(\Psi_{2}\right)\right| \leq K_{J}^{i}\left|\Psi_{1}-\Psi_{2}\right|
$$

and

$$
\left|h\left(t, \Psi_{1}\right)-h\left(t, \Psi_{2}\right)\right| \leq K_{\Psi}\left|\Psi_{1}-\Psi_{2}\right|,
$$

for all $\Psi_{1}, \Psi_{2} \in \mathbb{R}$ and $i=1,2,3, \ldots$.

Definition 3.1 A function $\Psi \in \Upsilon$ with its $\gamma$-derivative that exists on $[0, T]-\left\{t_{1}, t_{2}, t_{3}, \ldots\right.$, $\left.t_{m}\right\}$ is said to be the solution of the considered fractional impulsive problem under RBCs if it verifies the considered equation (1.1).

Lemma 3.1 Let $\Psi \in \Upsilon$ be the solution of the fractional impulsive problem with $\eta \in$ $C([0,1], \mathbb{R})$ given by

$$
\left\{\begin{array}{l}
D^{\gamma} \Psi(t)=\eta(t), \quad t \neq t_{i}, 1<\gamma \leq 2, \\
\delta \Psi\left(t_{j}\right)=I_{j}\left(\Psi\left(t_{j}\right)\right), \quad \delta \Psi^{\prime}\left(t_{j}\right)=J_{j}\left(\Psi\left(t_{j}\right)\right), \quad j=1,2,3, \ldots, l, \\
a \Psi(0)+b \Psi(T)=g_{1}(\Psi), \quad c \Psi^{\prime}(0)+d \Psi^{\prime}(T)=g_{2}(\Psi),
\end{array}\right.
$$


if and only is $\Psi$ is the solution of the fractional impulsive integral equation as

$$
\begin{aligned}
\Psi(t)= & \frac{1}{\Gamma(\gamma)} \int_{t_{k}}^{t}(t-s)^{\gamma-1} \eta(s) d s \\
& +\frac{1}{a+b}\left(\frac{a}{\Gamma(\gamma)} \sum_{j=1}^{k} \int_{t_{j}-1}^{t_{j}}\left(t_{j}-s\right)^{\gamma-1} \eta(s) d s-\frac{b}{\Gamma(\gamma)} \sum_{j=1}^{k} \int_{t_{k}-1}^{T}(T-s)^{\gamma-1} \eta(s) d s\right) \\
& +\left(\sum_{j=1}^{k}\left(t-t_{j}\right)+\frac{b d T}{(a+b)(c+d)}-\frac{b}{a+b} \sum_{j=1}^{k}\left(T-t_{j}\right)-\frac{t d}{c+d}\right) \\
& \times \frac{1}{\Gamma(\gamma-1)} \sum_{j=1}^{k} \int_{t_{j}-1}^{t_{j}}\left(t_{j}-s\right)^{\gamma-2} \eta(s) d s \\
& +\left(\frac{b d T}{(a+b)(c+d)}-\frac{t d}{c+d}\right) \frac{1}{\Gamma(\gamma-1)} \int_{t_{k}}^{T}(T-s)^{\gamma-2} \eta(s) d s+\frac{a}{a+b} \sum_{j=1}^{k} I_{j}\left(\Psi\left(t_{j}\right)\right) \\
& +\left(\sum_{j=1}^{k}\left(t-t_{j}\right)+\frac{b d T}{(a+b)(c+d)}-\frac{b}{a+b} \sum_{j=1}^{k}\left(T-t_{j}\right)-\frac{t d}{c+d}\right) \sum_{j=1}^{k} J_{j}\left(\Psi\left(t_{j}\right)\right) \\
& \times\left(\frac{b T}{(a+b)(c+d)}+\frac{t}{c+d}\right) g_{2}(\Psi)+\frac{g_{1}(\Psi)}{a+b} .
\end{aligned}
$$

Proof Let $\Psi$ is a solution of (3.1), then, for $\eta \in C([0,1], \mathbb{R}), t \in[0, T]$, and using Lemma 2.2 for the given problem, we have two constants $f_{0}, f_{1}$, that is,

$$
\begin{aligned}
& \Psi(t)=I^{\gamma} \eta(t)-f_{0}-f_{1} t, \quad t \in\left[0, t_{1}\right] \\
& \Psi(t)=\frac{1}{\Gamma(\gamma)} \int_{0}^{t_{1}}\left(t_{1}-s\right)^{\gamma-1} \eta(s) d s-f_{0}-f_{1} t, \quad t \in\left[0, t_{1}\right] .
\end{aligned}
$$

Differentiating we get

$$
\Psi^{\prime}(t)=\frac{1}{\Gamma(\gamma-1)} \int_{0}^{t_{1}}\left(t_{1}-s\right)^{\gamma-2} \eta(s) d s-f_{1}, \quad t \in\left[0, t_{1}\right] .
$$

Likewise, for $t \in\left(t_{1}, t_{2}\right]$, there are constants $\mathbf{d}_{0}, \mathbf{d}_{1}$ with

$$
\begin{aligned}
& \Psi(t)=I^{\gamma} \eta(t)-\mathbf{d}_{0}-\mathbf{d}_{1}\left(t-t_{1}\right), \quad t \in\left(t_{1}, t_{2}\right] \\
& \Psi(t)=\frac{1}{\Gamma(\gamma)} \int_{t_{1}}^{t}\left(t_{1}-s\right)^{\gamma-1} \eta(s) d s-\mathbf{d}_{0}-\mathbf{d}_{1}\left(t-t_{1}\right), \quad t \in\left[0, t_{1}\right] .
\end{aligned}
$$

Differentiating, we get

$$
\Psi^{\prime}(t)=\frac{1}{\Gamma(\gamma-1)} \int_{t_{1}}^{t}\left(t_{1}-s\right)^{\gamma-1} \eta(s) d s-\mathbf{d}_{1}, \quad t \in\left(t_{1}, t_{2}\right],
$$

and

$$
\begin{aligned}
& \Psi\left(t_{1}^{-}\right)=\frac{1}{\Gamma(\gamma)} \int_{0}^{t_{1}}\left(t_{1}-s\right)^{\gamma-1}-f_{0}-f_{1} t_{1}, \quad t \in\left[0, t_{1}\right], \\
& \Psi\left(t_{1}^{+}\right)=-f_{0}
\end{aligned}
$$




$$
\begin{aligned}
& \Psi^{\prime}\left(t_{1}^{-}\right)=\frac{1}{\Gamma(\gamma-1)} \int_{t_{1}}^{t}\left(t_{1}-s\right)^{\gamma-1} \eta(s) d s-\mathbf{d}_{1}, \quad t \in\left(t_{1}, t_{2}\right] \\
& \Psi^{\prime}\left(t_{1}^{+}\right)=-\mathbf{d}_{1} .
\end{aligned}
$$

Next applying the impulsive conditions after simplification we get the following:

$$
\begin{aligned}
& -\mathbf{d}_{0}=\frac{1}{\Gamma(\gamma)} \int_{0}^{t_{1}}\left(t_{1}-s\right)^{\gamma-1} \eta(s) d s-f_{0}-f_{1} t_{1}+I_{1}\left(\Psi\left(t_{1}\right)\right), \\
& -\mathbf{d}_{1}=\frac{1}{\Gamma(\gamma-1)} \int_{0}^{t_{1}}\left(t_{1}-s\right)^{\gamma-2} \eta(s) d s-f_{0}+J_{1}\left(\Psi\left(t_{1}\right)\right) .
\end{aligned}
$$

Putting these values in the given equation we get

$$
\begin{aligned}
\Psi(t)= & \frac{1}{\Gamma(\gamma)} \int_{0}^{t_{1}}\left(t_{1}-s\right)^{\gamma-1} \eta(s) d s+\frac{1}{\Gamma(\gamma)} \int_{0}^{t_{1}}\left(t_{1}-s\right)^{\gamma-1} \eta(s) d s \\
& +\frac{t-t_{1}}{\Gamma(\gamma-1)} \int_{0}^{t_{1}}\left(t_{1}-s\right)^{\gamma-1} \eta(s) d s \\
& +\left(t-t_{1}\right) J_{1}\left(\Psi\left(t_{1}\right)\right)+I_{1}\left(\Psi\left(t_{1}\right)\right)-f_{0}-f_{1} t .
\end{aligned}
$$

Reiterating the same procedure generally for $t \in\left(t_{j-1}, t_{j}\right]$, we have

$$
\begin{aligned}
\Psi(t)= & \frac{1}{\Gamma(\gamma)} \int_{0}^{t_{1}}\left(t_{1}-s\right)^{\gamma-1} \eta(s) d s+\frac{1}{\Gamma(\gamma)} \sum_{j=1}^{k} \int_{t_{j-1}}^{t_{j}}\left(t_{1}-s\right)^{\gamma-1} \eta(s) d s \\
& +\sum_{j=1}^{k}\left(t-t_{j}\right) \frac{1}{\Gamma(\gamma-1)} \int_{t_{j-1}}^{t_{j}}\left(t_{j}-s\right)^{\gamma-2} \eta(s) d s+\sum_{j=1}^{k}\left(t-t_{j}\right) J_{j}\left(\Psi\left(t_{j}\right)\right) \\
& +\sum_{j=1}^{k} I_{j}\left(\Psi\left(t_{j}\right)\right)-f_{0}-f_{1} t .
\end{aligned}
$$

In view of the RBCs and after simplification, we have the subsequent values for the constant $f_{0}, f_{1}$,

$$
\begin{aligned}
f_{0}= & \frac{b}{a+b}\left(\frac{1}{\Gamma(\gamma)} \int_{t_{k}}^{T}(T-s)^{\gamma-1} \eta(s) d s+\frac{1}{\Gamma(\gamma)} \sum_{j=1}^{k} \int_{t_{j-1}}^{t_{j}}\left(t_{j}-s\right)^{\gamma-1} \eta(s) d s\right. \\
& +\sum_{j=1}^{k}\left(T-t_{j}\right) \int_{t_{j-1}}^{t_{j}}\left(t_{j}-s\right)^{\gamma-2} \frac{1}{\Gamma(\gamma)} \eta(s) d s+\sum_{j=1}^{k}\left(T-t_{j}\right) J_{j}\left(\Psi\left(t_{j}\right)\right) \\
& \left.+\sum_{j=1}^{k}\left(T-t_{j}\right) I_{j}\left(\Psi\left(t_{j}\right)\right)\right)-\frac{b d T}{(a+b)(c+d)}\left(\frac{1}{\Gamma(\gamma-1)} \int_{t_{k}}^{T}(T-s)^{\gamma-2} \eta(s) d s\right. \\
& \left.+\frac{1}{\Gamma(\gamma-1)} \sum_{j=1}^{k} \int_{t_{j-1}}^{t_{j}}\left(t_{j}-s\right)^{\gamma-2} \eta(s) d s\right)-\frac{b d T}{(a+b)(c+d)} \sum_{j=1}^{k} J_{j}\left(\Psi\left(t_{j}\right)\right) \\
& +\left(\frac{b d T}{(a+b)(c+d)}\right) g_{2}\left(\Psi\left(t_{j}\right)\right)-\left(\frac{1}{a+b}\right) g_{1}\left(\Psi\left(t_{j}\right)\right),
\end{aligned}
$$




$$
\begin{aligned}
f_{1}= & \frac{d}{d+c}\left(\frac{1}{\Gamma(\gamma-1)} \int_{t_{k}}^{T}(T-s)^{\gamma-2} \eta(s) d s+\frac{1}{\Gamma(\gamma-1)} \sum_{j=1}^{k} \int_{t_{j}-1}^{t_{j}}\left(t_{j}-s\right)^{\gamma-2} \eta(s) d s\right. \\
& \left.+\sum_{j=1}^{k} J_{j}\left(\Psi\left(t_{j}\right)\right)\right)-\left(\frac{1}{d+c}\right) g_{2}\left(\Psi\left(t_{j}\right)\right) .
\end{aligned}
$$

Putting these values in (3.11), we get the required solution given in (3.2) as

$$
\begin{aligned}
\Psi(t)= & \frac{1}{\Gamma(\gamma)} \int_{t_{k}}^{t}(t-s)^{\gamma-1} \eta(s) d s \\
& +\frac{1}{\Gamma(\gamma)} \sum_{j=1}^{k} \int_{t_{j}-1}^{t_{j}}(t-s)^{\gamma-1} \eta(s) d s+\frac{1}{\Gamma(\gamma-1)} \sum_{j=1}^{k}\left(t-t_{j}\right) \int_{t_{j}-1}^{t_{j}}(t-s)^{\gamma-2} \eta(s) d s \\
& +\sum_{j=1}^{k}\left(t-t_{j}\right) J_{j}\left(\Psi\left(t_{j}\right)\right)+\sum_{j=1}^{k} I_{j}\left(\Psi\left(t_{j}\right)\right)-\frac{b}{a+b}\left(\frac{1}{\Gamma(\gamma)} \int_{t_{k}}^{T}(T-s)^{\gamma-1} \eta(s) d s\right. \\
& +\frac{1}{\Gamma(\gamma)} \sum_{j=1}^{k} \int_{t_{j-1}}^{t_{j}}\left(t_{j}-s\right)^{\gamma-1} \eta(s) d s+\sum_{j=1}^{k}\left(T-t_{j}\right) \frac{1}{\Gamma(\gamma-1)} \int_{t_{j-1}}^{t_{j}}\left(t_{j}-s\right)^{\gamma-2} \eta(s) d s \\
& \left.+\sum_{j=1}^{k}\left(T-t_{j}\right) J_{j}\left(\Psi\left(t_{j}\right)\right)+\sum_{j=1}^{k} I_{j}\left(\Psi\left(t_{j}\right)\right)\right) \\
& +\frac{b d T}{(a+b)(c+d)}\left(\frac{1}{\Gamma(\gamma-1)} \int_{t_{k}}^{T}(T-s)^{\gamma-2} \eta(s) d s\right. \\
& \left.+\frac{1}{\Gamma(\gamma-1)} \sum_{j=1}^{k} \int_{t_{j}-1}^{t_{j}}\left(t_{j}-s\right)^{\gamma-2} \eta(s) d s+\sum_{j=1}^{k} J_{j}\left(\Psi\left(t_{j}\right)\right)+\frac{g_{2}(\Psi)}{d}\right) \\
& +\frac{t d}{d+c}\left(\frac{1}{\Gamma(\gamma-1)} \int_{t_{k}}^{T}(T-s)^{\gamma-2} \eta(s) d s\right. \\
& \left.+\frac{1}{\Gamma(\gamma-1)} \sum_{j=1}^{k} \int_{t_{j}-1}^{t_{j}}\left(t_{j}-s\right)^{\gamma-2} \eta(s) d s+\sum_{j=1}^{k} J_{j}\left(\Psi\left(t_{j}\right)\right)\right) \\
& +\left(\frac{t}{d+c}\right) g_{2}(\Psi)+\left(\frac{1}{a+b}\right) g_{1}(\Psi) . \\
&
\end{aligned}
$$

Rearranging terms so we can achieve the solution given in (3.2). On the other hand, we presume that $\Psi$ is a solution of the given equation, it being straightforward we give the solution given by (3.2), by direct computation which satisfies (3.12).

Corollary 3.1 In view of Lemma 3.1, the solution of the considered problem (1.1) is given by

$$
\begin{aligned}
\Psi(t)= & \frac{1}{\Gamma(\gamma)} \int_{t_{k}}^{t}(t-s)^{\gamma-1} h(s, \Psi(s)) d s+\frac{a}{(a+b) \Gamma(\gamma)} \sum_{j=1}^{k} \int_{t_{j}-1}^{t_{j}}\left(t_{j}-s\right)^{\gamma-1} h(s, \Psi(s)) d s \\
& -\frac{b}{(a+b) \Gamma(\gamma)} \int_{t_{k}}^{T}(T-s)^{\gamma-1} h(s, \Psi(s)) d s+\left(\sum_{j=1}^{k}\left(t-t_{j}\right)+\frac{b d T}{(a+b)(c+d)}\right.
\end{aligned}
$$




$$
\begin{aligned}
& \left.-\frac{b}{a+b} \sum_{j=1}^{k}\left(T-t_{j}\right)-\frac{t d}{c+d}\right) \frac{1}{\Gamma(\gamma-1)} \sum_{j=1}^{k} \int_{t_{j}-1}^{t_{j}}\left(t_{j}-s\right)^{\gamma-2} h(s, \Psi(s)) d s \\
& +\left(\frac{b d T}{(a+b)(c+d)}-\frac{t d}{c+d}\right) \frac{1}{\Gamma(\gamma-1)} \int_{t_{k}}^{T}(T-s)^{\gamma-2} h(s, \Psi(s)) d s \\
& +\frac{a}{a+b} \sum_{j=1}^{k} I_{j}\left(\Psi\left(t_{j}\right)\right)+\left(\sum_{j=1}^{k}\left(t-t_{j}\right)+\frac{b d T}{(a+b)(c+d)}-\frac{b}{a+b} \sum_{j=1}^{k}\left(T-t_{j}\right)\right. \\
& \left.-\frac{t d}{c+d}\right) \sum_{j=1}^{k} J_{j}\left(\Psi\left(t_{j}\right)\right)+\left(\frac{b T}{(a+b)(c+d)}+\frac{t}{c+d}\right) g_{2}(\Psi)+\frac{g_{1}(\Psi)}{a+b} .
\end{aligned}
$$

With the help of Corollary 3.1, the problem (3.12) is reduced to the fixed point problem as $\Psi=T(\Psi)$. Keeping in mind this operator problem we develop results regarding the solution of the considered problem.

Next we define five operators to establish the main results as regards the existence and uniqueness as follows:

$$
\begin{aligned}
F_{0}: \Upsilon \rightarrow & \Upsilon \\
F_{0} \Psi(t)= & \frac{a}{a+b} \sum_{j=1}^{k} I_{j}\left(\Psi\left(t_{j}\right)\right) \\
& +\left(\sum_{j=1}^{k}\left(t-t_{j}\right)+\frac{b d T}{(a+b)(c+d)}-\frac{b}{a+b} \sum_{j=1}^{k}\left(T-t_{j}\right)-\frac{t d}{c+d}\right) \sum_{j=1}^{k} J_{j}\left(\Psi\left(t_{j}\right)\right) \\
& +\left(\frac{b T}{(a+b)(c+d)}+\frac{t}{c+d}\right) g_{2}(\Psi)+\frac{g_{1}(\Psi)}{a+b} .
\end{aligned}
$$

For the integral part we define the operators by

$$
\begin{aligned}
& F_{1}: \Upsilon \rightarrow \Upsilon \\
& F_{1} \Psi(t)=\frac{1}{\Gamma(\gamma)} \int_{t_{k}}^{t}(t-s)^{\gamma-1} h(s, \Psi(s)) d s-\frac{b}{(a+b) \Gamma(\gamma)} \int_{t_{k}}^{T}(T-s)^{\gamma-1} h(s, \Psi(s)) d s, \\
& F_{2}: \Upsilon \rightarrow \Upsilon \\
& F_{2} \Psi(t)=\left(\sum_{j=1}^{k}\left(t-t_{j}\right)+\frac{b d T}{(a+b)(c+d)}-\frac{b}{a+b} \sum_{j=1}^{k}\left(T-t_{j}\right)-\frac{t d}{c+d}\right) \\
& \times \frac{1}{\Gamma(\gamma-1)} \sum_{j=1}^{k} \int_{t_{j}-1}^{t_{j}}\left(t_{j}-s\right)^{\gamma-2} h(s, \Psi(s)) d s, \\
& F_{3}: \Upsilon \rightarrow \Upsilon \\
& F_{3} \Psi(t)=\left(\frac{b d T}{(a+b)(c+d)}-\frac{t d}{c+d}\right) \frac{1}{\Gamma(\gamma-1)} \int_{t_{k}}^{T}(T-s)^{\gamma-2} h(s, \Psi(s)) d s, \\
& F_{4}: \Upsilon \rightarrow \Upsilon \\
& F_{4} \Psi(t)=\frac{a}{(a+b) \Gamma(\gamma)} \sum_{j=1}^{k} \int_{t_{j}-1}^{t_{j}}\left(t_{j}-s\right)^{\gamma-1} h(s, \Psi(s)) d s .
\end{aligned}
$$


Let $T: \Upsilon \rightarrow \Upsilon$, then $T$ is defined by

$$
T \Psi(t)=F_{0} \Psi(t)+F_{1} \Psi(t)+F_{2} \Psi(t)+F_{3} \Psi(t)+F_{4} \Psi(t)
$$

Therefore, investigating the solution to the given (3.12) the problem is just like investigating the fixed point for the operator $T$.

Theorem 3.1 The operator $F_{0}: \Upsilon \rightarrow \Upsilon$ is Lipschitz with constant $K_{I}=\sum_{i=1}^{m} K_{I}^{i} \in[0,1)$. Consequently $F_{0}$ is $\psi$-Lipschitz with the same constant $K_{I}^{i} \in[0,1)$. Further $F_{0}$ verifies the following relation:

$$
\left\|F_{0} \Psi\right\| \leq A+B\|\Psi\|,
$$

where

$$
\begin{aligned}
A= & \left|\frac{a}{a+b}\right| M_{1}+\left|l T+\frac{b d t}{(a+b)(c+d)}\right| M_{2} \\
& +\left|\frac{b T}{(a+b)(c+d)}+\frac{T}{c+d}\right| M_{g_{2}}+\left|\frac{a}{a+b}\right| M_{g_{1}}
\end{aligned}
$$

and

$$
\begin{aligned}
B= & \left|\frac{a}{a+b}\right| C_{1}+\left|l T+\frac{b d t}{(a+b)(c+d)}\right| C_{2} \\
& +\left|\frac{b T}{(a+b)(c+d)}+\frac{T}{c+d}\right| C_{g_{2}}+\left|\frac{a}{a+b}\right| C_{g_{1}} .
\end{aligned}
$$

Proof Using $\left(A_{1}\right)$ and $\left(A_{4}\right)$, we have

$$
\begin{aligned}
& \sup _{t \in[0, T]}\left|F_{0} \Psi_{1}-F_{0} \Psi_{2}\right| \\
& =\sup _{t \in[0, T] \mid} \mid \frac{a}{a+b} \sum_{j=1}^{k}\left(I_{j}\left(\Psi_{1}\left(t_{j}\right)\right)-I_{j}\left(\Psi_{2}\left(t_{j}\right)\right)\right)+\left(\sum_{j=1}^{k}\left(t-t_{j}\right)+\frac{b d T}{(a+b)(c+d)}\right. \\
& \left.\quad-\frac{b}{a+b} \sum_{j=1}^{k}\left(T-t_{j}\right)-\frac{t d}{(c+d)}\right)\left[J_{j}\left(\Psi_{1}\left(t_{j}\right)\right)-J_{j}\left(\Psi_{2}\left(t_{j}\right)\right)\right]+\left(\frac{b T}{(a+b)(c+d)}\right. \\
& \left.\quad+\frac{t}{c+d}\right)\left(g_{2}\left(\Psi_{1}\right)-g_{2}\left(\Psi_{2}\right)\right)+\frac{1}{a+b}\left(g_{1}\left(\Psi_{1}\right)-g_{1}\left(\Psi_{2}\right)\right) \mid .
\end{aligned}
$$

Further simplification yields

$$
\begin{aligned}
\left\|F_{0} \Psi_{1}-F_{0} \Psi_{2}\right\| \leq & \left|\frac{a}{a+b}\right| K_{1}^{i}\left\|\Psi_{1}-\Psi_{2}\right\|+\left|l T+\frac{b d T}{(a+b)(c+d)}\right| K_{j}^{i}\left\|\Psi_{1}-\Psi_{2}\right\| \\
& +\left|\frac{b T}{(a+b)(c+d)}+\frac{T}{c+d}\right| k_{g_{2}}\left\|\Psi_{1}-\Psi_{2}\right\|+\left|\frac{1}{a+b}\right| K_{g_{1}}\left\|\Psi_{1}-\Psi_{2}\right\| .
\end{aligned}
$$


Hence one has

$$
\begin{aligned}
\left\|F_{0} \Psi_{1}-F_{0} \Psi_{2}\right\| \leq & \left(\left|\frac{a}{a+b}\right| K_{1}^{i}+\left|l T+\frac{b d T}{(a+b)(c+d)}\right| K_{j}^{i}+\left|\frac{b T}{(a+b)(c+d)}+\frac{T}{c+d}\right| k_{g_{2}}\right. \\
& \left.+\left|\frac{1}{a+b}\right| K_{g_{1}}\right) \times\left\|\Psi_{1}-\Psi_{2}\right\| .
\end{aligned}
$$

Using

$$
\begin{aligned}
K_{1}= & \left(\left|\frac{a}{a+b}\right| K_{1}^{i}+\left|l T+\frac{b d T}{(a+b)(c+d)}\right| K_{j}^{i}+\left|\frac{b T}{(a+b)(c+d)}+\frac{T}{c+d}\right| k_{g_{2}}\right. \\
& \left.+\left|\frac{1}{a+b}\right| K_{g_{1}}\right) .
\end{aligned}
$$

Then (3.16) becomes

$$
\left\|F_{0} \Psi_{1}-F_{0} \Psi_{2}\right\| \leq K_{1}\left\|\Psi_{1}-\Psi_{2}\right\|
$$

Thus $F_{0}$ is Lipschitz with constant $K \in[0,1)$.

For the growth relation using $\left(A_{2}\right)$ and $\left(A_{5}\right)$, we get the following:

$$
\begin{aligned}
\left\|F_{0} \Psi\right\|= & \sup _{t \in[0, T]} \mid \frac{a}{a+b} \sum_{j=1}^{k} I_{j}\left(\Psi_{1}\left(t_{j}\right)\right)+\left(\sum_{j=1}^{k}\left(t-t_{j}\right)+\frac{b d T}{(a+b)(c+d)}\right. \\
& \left.-\left(\frac{b}{a+b}\right) \sum_{j=1}^{k}\left(T-t_{j}\right)-\frac{t d}{(c+d)}\right) J_{j}\left(\Psi\left(t_{j}\right)\right) \\
& +\left(\frac{b T}{(a+b)(c+d)}+\frac{t}{c+d}\right) g_{2}(\Psi)+\left(\frac{1}{a+b}\right) g_{1}(\Psi) \mid, \\
\left\|F_{0} \Psi\right\| \leq & \left|\frac{a}{a+b}\right|\left(C_{1}\|\Psi\|+M_{1}\right)+\left|l T+\frac{b d T}{(a+b)(c+d)}\right|\left(C_{2}\|\Psi\|+M_{2}\right) \\
& +\left|\frac{b T}{(a+b)(c+d)}+\frac{T}{c+d}\right|\left(C_{g_{2}}\|\Psi\|+M_{g_{2}}\right) \\
& +\left|\frac{1}{a+b}\right|\left(C_{g_{1}}\|\Psi\|+M_{g_{1}}\right) .
\end{aligned}
$$

Then (3.17) becomes

$$
\left\|\left|F_{0} \Psi(t)\right|\right\| \leq A+B\|\Psi\|
$$

where $A$ and $B$ are given in (3.14) and (3.15).

Lemma 3.2 The operator

$F_{1} \Psi(t): \Upsilon \rightarrow \Upsilon$ 
is continuous and satisfies the following growth condition:

$$
\left\|F_{1} \Psi\right\| \leq\left(1+\left|\frac{b}{(a+b)}\right|\right) \frac{T^{\gamma}}{\Gamma(\gamma+1)}\left(C_{f}+M_{f}\|\Psi\|\right) .
$$

Proof Let $\Psi_{n}$ be a sequence in $B_{k}=\{\Psi \in Y:\|\Psi\| \leq r\}$ such that $\Psi_{n} \rightarrow \Psi$ as $n \rightarrow \infty$.

This implies that

$$
\frac{(t-s)^{\gamma-1}}{\Gamma(\gamma)}\left(h\left(s, \Psi_{n}\right)-h(s, \Psi)\right) \rightarrow 0, \quad n \rightarrow \infty,
$$

and

$$
\frac{(T-s)^{\gamma-1}}{\Gamma(\gamma)}\left(h\left(s, \Psi_{n}\right)-h(s, \Psi)\right) \rightarrow 0, \quad n \rightarrow \infty .
$$

Using the Lebesgue dominated convergence theorem, $\left\|F_{1}\left(\Psi_{n}\right)-F_{1}(\Psi)\right\| \rightarrow 0$ as $n \rightarrow \infty$, this implies that $F_{1}$ is continuous.

For the growth relation, using $\left(A_{3}\right)$, we then find

$$
\begin{aligned}
&\left|F_{1}(\Psi(t))\right| \leq \frac{1}{\Gamma(\gamma)} \int_{t_{k}}^{t}(t-s)^{\gamma-1}|h(s, \Psi(s))| d s \\
&+\left|\frac{b}{a+b}\right| \frac{1}{\Gamma(\gamma)} \int_{t_{k}}^{T}(T-s)^{\gamma-1}|h(s, \Psi(s))| d s, \\
& \sup _{t \in[0, T]}\left|F_{1}(\Psi(t))\right| \leq \sup _{t \in[0, T]}\left(\frac{\left(t-t_{k}\right)^{\gamma}}{\Gamma(\gamma+1)}+\left|\frac{b}{a+b}\right| \frac{\left(T-t_{k}\right)^{\gamma}}{\Gamma(\gamma+1)}\right)\left(C_{f}+M_{f}\|\Psi\|\right), \\
&\left\|F_{1} \Psi\right\| \leq\left(1+\left\|\frac{b}{a+b}\right\|\right) \frac{T^{\gamma}}{\Gamma(\gamma+1)}\left(C_{f}+M_{f}\|\Psi\|\right) .
\end{aligned}
$$

Theorem 3.2 The operator defined as $F_{1}: Y \rightarrow Y$ is compact and $\psi$-Lipschitz with constant zero.

Proof Clearly $F_{1}$ satisfies the growth condition so $F_{1}$ is bounded on $B_{k}=\{\Psi \in Y:\|\Psi\| \leq$ $r\}$.

Let $\Psi \in B_{k}$, we have

$$
\left\|F_{1} \Psi\right\| \leq\left(1+\left|\frac{b}{a+b}\right|\right) \frac{T^{\gamma}}{\Gamma(\gamma+1)}\left(C_{f}+M_{f}\|\Psi\|\right) \leq Q_{1} .
$$

Hence $F_{1}$ is bounded.

Further for $0 \leq t_{1} \leq t_{2} \leq T$, we show $F_{1}$ is equi-continuous.

$$
\begin{aligned}
& \left|F_{1}\left(\Psi\left(t_{1}\right)\right)-F_{1}\left(\Psi\left(t_{2}\right)\right)\right| \\
& \leq \frac{1}{\Gamma(\gamma)} \int_{t_{k}}^{t_{1}}\left(t_{1}-s\right)^{\gamma-1}|h(s, \Psi(s))| d s-\frac{1}{\Gamma(\gamma)} \int_{t_{k}}^{t_{2}}\left(t_{2}-s\right)^{\gamma-1}|h(s, \Psi(s))| d s \\
& \quad+\frac{b}{a+b} \frac{1}{\Gamma(\gamma)} \int_{t_{k}}^{T}(T-s)^{\gamma-1}|h(s, \Psi(s))| d s \\
& \quad-\frac{b}{a+b} \frac{1}{\Gamma(\gamma)} \int_{t_{k}}^{T}(T-s)^{\gamma-1}|h(s, \Psi(s))| d s .
\end{aligned}
$$


One has

$$
\left|F_{1}\left(\Psi\left(t_{1}\right)\right)-F_{1}\left(\Psi\left(t_{2}\right)\right)\right| \leq \frac{\left(C_{f}+M_{f}\|\Psi\|\right)}{\Gamma(\gamma+1)}\left(\left(t_{1}-t_{k}\right)^{\gamma}-\left(t_{2}-t_{k}\right)^{\gamma}\right) .
$$

As $t_{1} \rightarrow t_{2}$, then $\left|F_{1}\left(\Psi\left(t_{1}\right)\right)-F_{1}\left(\Psi\left(t_{2}\right)\right)\right| \rightarrow 0$. Hence $F_{1}$ is equi-continuous so $F_{1}$ is compact.

Then, by Proposition 2.3, $F_{1}$ is $\psi$-Lipschitz with constant zero.

Lemma 3.3 The operator

$$
F_{2}: \Upsilon \rightarrow \Upsilon
$$

is continuous and satisfies the following growth condition:

$$
\left\|F_{2} \Psi\right\| \leq\left(l+\left|\frac{b d}{(a+b)(c+d)}\right|\right) \frac{T^{\gamma+1}}{\Gamma(\gamma)}\left(C_{f}+M_{f}\|\Psi\|\right) .
$$

Proof Let $\Psi_{n}$ be a sequence in $B_{k}=\{\Psi \in Y:\|\Psi\| \leq r\}$ such that $\Psi_{n} \rightarrow \Psi$ as $n \rightarrow \infty$.

This implies that

$$
\frac{\left(t_{i}-s\right)^{\gamma-2}}{\Gamma(\gamma-1)}\left(h\left(s, \Psi_{n}\right)-h(s, \Psi)\right) \rightarrow 0, \quad n \rightarrow \infty,
$$

Using the Lebesgue dominated convergence theorem, we see that

$$
\left\|F_{2}\left(\Psi_{n}\right)-F_{2}(\Psi)\right\| \rightarrow 0 \text { as } n \rightarrow \infty .
$$

This implies that $F_{2}$ is continuous.

Further for the growth relation using $\left(A_{3}\right)$, then one has

$$
\begin{aligned}
\left|F_{2}(\Psi(t))\right| \leq & \left|\sum_{j=1}^{k}\left(t-t_{j}\right)+\frac{b d T}{(a+b)(c+d)}-\frac{b}{a+b} \sum_{j=1}^{k}\left(T-t_{j}\right)-\frac{t d}{c+d}\right| \\
& \times \sum_{j=1}^{k} \int_{t_{j}-1}^{t_{j}} \frac{\left(t_{j}-s\right)^{\gamma-2}}{\Gamma(\gamma)}|h(s, \Psi(s))| d s,
\end{aligned}
$$

which yields

$$
\sup _{t \in[0, T]}\left|F_{2}(\Psi(t))\right| \leq \sup _{t \in[0, T]}\left|\sum_{j=1}^{k}\left(t-t_{j}\right)+\frac{b d T}{(a+b)(c+d)}\right| \times \int_{t_{j}-1}^{t_{j}} \frac{\left(t_{j}-s\right)^{\gamma-2}}{\Gamma(\gamma)}|h(s, \Psi(s))| d s .
$$

Thus one has

$$
\left\|F_{2}(\Psi)\right\| \leq\left|l+\frac{b d}{(a+b)(c+d)}\right| \times \frac{T^{\gamma+1}}{\Gamma(\gamma)}\left(C_{f}+M_{f}\|\Psi\|\right) .
$$

This is the required relation.

Lemma 3.4 The operator defined as $F_{2}: Y \rightarrow Y$ is compact and $F_{2}$ is $\psi$-Lipschitz with constant zero. 
Proof It can be easily derived similarly to Lemma 3.3.

Lemma 3.5 The operator

$$
F_{3}: \Upsilon \rightarrow \Upsilon
$$

is continuous and satisfies the following growth condition:

$$
\left\|F_{3} \Psi\right\| \leq\left|\frac{b d}{(a+b)(c+d)}\right| \times \frac{T^{\gamma+1}}{\Gamma(\gamma)}\left(C_{f}+M_{f}\|\Psi\|\right) .
$$

Proof Let $\Psi_{n}$ be a sequence in $B_{k}=\{\Psi \in Y:\|\Psi\| \leq r\}$ such that $\Psi_{n} \rightarrow \Psi$ as $n \rightarrow \infty$.

This implies that

$$
\frac{(T-s)^{\gamma-2}}{\Gamma(\gamma-1)}\left(h\left(s, \Psi_{n}\right)-h(s, \Psi)\right) \rightarrow 0, \quad n \rightarrow \infty .
$$

Using the Lebesgue dominated convergence theorem

$$
\left\|F_{3}\left(\Psi_{n}\right)-F_{3}(\Psi)\right\| \rightarrow 0 \quad \text { as } n \rightarrow \infty .
$$

This implies that $F_{3}$ is continuous.

For the growth relation using $\left(A_{3}\right)$, then

$$
\begin{aligned}
& \left|F_{3}(\Psi(t))\right| \leq\left|\frac{b d T}{(a+b)(c+d)}-\frac{t d}{c+d}\right| \times \frac{1}{\Gamma(\gamma-1)} \int_{t_{k}}^{T}(T-s)^{\gamma-2}|h(s, \Psi(s))| d s, \\
& \sup _{t \in[0, T]}\left|F_{3}(\Psi(t))\right| \leq \sup _{t \in[0, T]}\left|\frac{b d T}{(a+b)(c+d)}\right| \times \int_{t_{k}}^{T}(T-s)^{\gamma-2}|h(s, \Psi(s))| d s .
\end{aligned}
$$

Hence one has

$$
\left\|F_{3}(\Psi)\right\| \leq\left|\frac{b d}{(a+b)(c+d)}\right| \times \frac{T^{\gamma+1}}{\Gamma(\gamma)}\left(C_{f}+M_{f}\|\Psi\|\right) .
$$

This is the required relation.

Lemma 3.6 The operator defined by $F_{3}: Y \rightarrow Y$ is compact and $F_{3}$ is $\psi$-Lipschitz with constant zero.

Proof The proof is so easy that we omit it.

\section{Lemma 3.7 The operator}

$$
F_{4}: \Upsilon \rightarrow \Upsilon
$$

is continuous and satisfies the following growth condition:

$$
\left\|F_{4} \Psi\right\| \leq\left|\frac{a}{a+b}\right| \times \frac{l T^{\gamma}}{\Gamma(\gamma+1)}\left(C_{f}+M_{f}\|\Psi\|\right) .
$$


Proof Let $\Psi_{n}$ be a sequence in $B_{k}=\{\Psi \in Y:\|\Psi\| \leq r\}$ such that $\Psi_{n} \rightarrow \Psi$ as $n \rightarrow \infty$.

This implies that

$$
\frac{\left(t_{j}-s\right)^{\gamma-1}}{\Gamma(\gamma)}\left(h\left(s, \Psi_{n}\right)-h(s, \Psi)\right) \rightarrow 0, \quad n \rightarrow \infty,
$$

Using the Lebesgue dominated convergence theorem, $\left\|F_{4}\left(\Psi_{n}\right)-F_{4}(\Psi)\right\| \rightarrow 0$ as $n \rightarrow \infty$. This implies that $F_{4}$ is continuous.

For the growth relation using $\left(A_{3}\right)$, then

$$
\begin{aligned}
& \left|F_{4}(\Psi(t))\right| \leq\left|\frac{a}{a+b}\right| \frac{1}{\Gamma(\gamma)} \sum_{j=1}^{k} \int_{t_{j-1}}^{t_{j}}\left(t_{j}-s\right)^{\gamma-1}|h(s, \Psi(s))| d s, \\
& \sup _{t \in[0, T]}\left|F_{4}(\Psi(t))\right| \leq \sup _{t \in[0, T]}\left|\frac{a}{a+b}\right| \times \frac{\left(t_{j}-t_{j-1}\right)^{\gamma}}{\Gamma(\gamma+1)} \times\left(C_{f}+M_{f}\|\Psi\|\right), \\
& \left\|F_{4} \Psi\right\| \leq\left|\frac{a}{a+b}\right| \times \frac{l T^{\gamma}}{\Gamma(\gamma+1)}\left(C_{f}+M_{f}\|\Psi\|\right) .
\end{aligned}
$$

This is the required relation.

Lemma 3.8 The operator defined by $F_{4}: Y \rightarrow Y$ is compact and $F_{4}$ is $\psi$-Lipschitz with constant zero.

Proof The proof is so easy that we omit it.

Next we show in combined form thta the four operators $F_{1}, F_{2}, F_{3}$ and $F_{4}$, satisfy the growth condition and are continuous, then we show the operators $F_{1}, F_{2}, F_{3}$ and $F_{4}$ are $\psi$-Lipschitz with constants zero and co-mpact.

Theorem 3.3 The operators $F_{1}, F_{2}, F_{3}$ and $F_{4}: \Upsilon \rightarrow \Upsilon$, are continuous and satisfy the following relation:

$$
\begin{aligned}
& \left\|F_{1} \Psi\right\|+\left\|F_{2} \Psi\right\|+\left\|F_{3} \Psi\right\|+\left\|F_{4} \Psi\right\| \\
& \leq \\
& \quad+\left|\frac{b}{(a+b)}\right| \frac{T^{\gamma}}{\Gamma(\gamma+1)}+\left|l+\frac{b d}{(a+b)(c+d)}\right| \frac{T^{\gamma+1}}{\Gamma(\gamma)} \\
& \quad \times\left(C_{f}+M_{f}\|\Psi\|\right) .
\end{aligned}
$$

Since $F_{1}, F_{2}, F_{3}$ and $F_{4}$, are continuous, $F_{1}+F_{2}+F_{3}+F_{4}$, is continuous.

Lemma 3.9 The operators $F_{1}, F_{2}, F_{3}$ and $F_{4}: \Upsilon \rightarrow \Upsilon$, are compact and $\psi$-Lipschitz with constant zero.

Proof Since $F_{1}, F_{2}, F_{3}$ and $F_{4}$, are compact, $F_{1}+F_{2}+F_{3}+F_{4}$, is compact, then, by Proposition 2.3, it is also $\psi$-Lipschitz with zero constant. 
Theorem 3.4 Presume that $\left(A_{1}\right),\left(A_{2}\right),\left(A_{3}\right)$ and $\left(A_{4}\right)$, hold, then the problem 3.2 has at least one solution $\Psi \in \Upsilon$ and the set of the solutions of the problem 3.2 is bounded in $\Upsilon$.

Proof Let $F_{0}, F_{1}, F_{2}, F_{3}$ and $F_{4}, T: \Upsilon \rightarrow \Upsilon$ be the operators defined in the section above. They are bounded and continuous. Furthermore, $F_{0}$ is $\psi$-Lipschitz with constant $K \in[0,1)$ and $F_{1}, F_{2}, F_{3}$ and $F_{4}$ are $\psi$-Lipschitz with zero constants. Let

$$
H=\{\Psi \in \Upsilon: \text { there exist } \lambda \in[0,1] \text { such that } \Psi=\lambda T \Psi\}
$$

We show that $H$ is bounded in $\Upsilon$. Let $\Psi \in H, \lambda \in[0,1]$ such that $\|\Psi\|=\lambda\|T \Psi\|$. It follows from (3.13) and (3.22) that

$$
\begin{aligned}
\|\Psi\| \leq & |\lambda|\left(\left\|F_{0} \Psi\right\|+\left\|F_{1} \Psi\right\|+\left\|F_{2} \Psi\right\|+\left\|F_{3} \Psi\right\|+\left\|F_{4} \Psi\right\|\right), \\
\|\Psi\| \leq & |\lambda|\left[A+B\|\Psi\|+\left(1+\left|\frac{b}{a+b}\right|\right) \frac{T^{\gamma}}{\Gamma(\gamma+1)}+\left(l+\left|\frac{b d}{(a+b)(c+d)}\right|\right) \frac{T^{\gamma+1}}{\Gamma(\gamma)}\right. \\
& \left.+\left|\frac{b d}{(a+b)(c+d)}\right| \frac{T^{\gamma+1}}{\Gamma(\gamma)}+\left|\frac{a}{a+b}\right| \times \frac{1 T^{\gamma}}{\Gamma(\gamma+1)}\right]\left(M_{f}\|\Psi\|+C_{f}\right) .
\end{aligned}
$$

This inequality shows that $H$ is bounded; if not, then we assume that $\zeta=\|\Psi\| \rightarrow \infty$. Dividing both sides of the above inequality by $\|\Psi\|$, we have

$$
\begin{aligned}
1 \leq & |\lambda|\left[\frac{A+B\|\Psi\|+\left(1+\left|\frac{b}{a+b}\right|\right) \frac{T^{\gamma}}{\Gamma(\gamma+1)}+\left(l+\left|\frac{b d}{(a+b)(c+d)}\right|\right) \frac{T^{\gamma+1}}{\Gamma(\gamma)}}{\zeta}\right. \\
& \left.+\frac{\left|\frac{b d}{(a+b)(c+d)}\right| \frac{T^{\gamma+1}}{\Gamma(\gamma)}+\left|\frac{a}{a+b}\right| \times \frac{l T^{\gamma}}{\Gamma(\gamma+1)}}{\zeta}\right] \times\left(M_{f}\|\Psi\|+C_{f}\right) .
\end{aligned}
$$

Taking the limit as $\zeta \rightarrow \infty$, we get the relation $1 \leq 0$, which is not possible. Then we have the possibility that $T$ is bounded in $\Upsilon$ and has at least one fixed point.

Theorem 3.5 Under the hypothesis $\left(A_{5}\right)$ and if $\mathbf{K}<1$ holds, where

$$
\begin{aligned}
\mathbf{K}= & {\left[\left(\frac{T^{\gamma}}{\Gamma(\gamma+1)}+\left|\frac{a}{a+b}\right| \times \frac{l T^{\gamma}}{\Gamma(\gamma+1)}+\left|\frac{b}{a+b}\right| \times \frac{T^{\gamma}}{\Gamma(\gamma+1)}+\mid l\right.\right.} \\
& \left.+\frac{b d}{(a+b)(c+d)}\left|\times \frac{l T^{\gamma+1}}{\Gamma(\gamma)}+\right| \frac{b d}{(a+b)(c+d)} \mid \times \frac{T^{\gamma}}{\Gamma(\gamma)}\right) K_{\Psi}+\left|\frac{a}{a+b}\right| K_{I}^{i} \\
& \left.+\left|l+\frac{b d}{(a+b)(c+d)}\right| K_{J}^{i}+\left|\frac{b T}{(a+b)(c+d)}+\frac{1}{c+d}\right| K_{g_{2}}+\left|\frac{1}{a+b}\right| K_{g_{1}}\right],
\end{aligned}
$$

the considered problem (1.1) has a unique solution.

Proof Consider $\Psi_{1}, \Psi_{2} \in \Upsilon$ to be two solutions of the given problem (1.1), then

$$
\begin{aligned}
\left\|T \Psi_{1}-T \Psi_{2}\right\| \leq & {\left[\frac{K_{\Psi} T^{\gamma}}{\Gamma(\gamma+1)}+\left|\frac{a}{a+b}\right| \times \frac{K_{\Psi} l T^{\gamma}}{\Gamma(\gamma+1)}+\left|\frac{b}{a+b}\right| \times \frac{K_{\Psi} T^{\gamma}}{\Gamma(\gamma+1)}\right.} \\
& +\left|l+\frac{b d}{(a+b)(c+d)}\right| \times \frac{K_{\Psi} l T^{\gamma+1}}{\Gamma(\gamma)}+\left|\frac{b d}{(a+b)(c+d)}\right| \times \frac{K_{\Psi} T^{\gamma}}{\Gamma(\gamma)}
\end{aligned}
$$




$$
\begin{aligned}
& +\left|\frac{a}{a+b}\right| K_{I}^{i}+\left|l+\frac{b d}{(a+b)(c+d)}\right| K_{J}^{i} \\
& \left.+\left|\frac{b T}{(a+b)(c+d)}+\frac{1}{c+d}\right| K_{g_{2}}+\left|\frac{1}{a+b}\right| K_{g_{1}}\right]\left\|\Psi_{1}-\Psi_{2}\right\|, \\
\left\|T \Psi_{1}-T \Psi_{2}\right\| \leq & {\left[\left(\frac{T^{\gamma}}{\Gamma(\gamma+1)}+\left|\frac{a}{a+b}\right| \times \frac{l T^{\gamma}}{\Gamma(\gamma+1)}+\left|\frac{b}{a+b}\right| \times \frac{T^{\gamma}}{\Gamma(\gamma+1)}\right.\right.} \\
& \left.+\left|l+\frac{b d}{(a+b)(c+d)}\right| \times \frac{l T^{\gamma+1}}{\Gamma(\gamma)}+\left|\frac{b d}{(a+b)(c+d)}\right| \times \frac{T^{\gamma}}{\Gamma(\gamma)}\right) K_{\Psi} \\
& +\left|\frac{a}{a+b}\right| K_{I}^{i}+\left|l+\frac{b d}{(a+b)(c+d)}\right| K_{J}^{i}+\left|\frac{b T}{(a+b)(c+d)}+\frac{1}{c+d}\right| K_{g_{2}} \\
& \left.+\left|\frac{1}{a+b}\right| K_{g_{1}}\right]\left\|\Psi_{1}-\Psi_{2}\right\| .
\end{aligned}
$$

Thus $T$ is a contraction mapping and by the Banach contraction theorem $T$ has a unique fixed point. Thus the considered problem has a unique solution.

\section{Test problem}

Example 4.1 Let $\gamma=\frac{3}{2}, T=1, a=b=1, c=d=0.4$, and the subsequent problem be

$$
\left\{\begin{array}{l}
D^{\frac{3}{2}} \Psi(t)=\frac{\sin (t)}{16+|\Psi(t)|}, \quad t \in[0,1], t \neq \frac{1}{5}, \\
\delta \Psi\left(\frac{1}{5}\right)=I_{j}\left(\Psi\left(\frac{1}{5}\right)\right)=\frac{4+|\Psi|^{2}}{30+||^{2}}, \\
\delta \Psi^{\prime}\left(\frac{1}{5}\right)=J_{j}\left(\Psi^{\prime}\left(\frac{1}{5}\right)\right)=\frac{20+|\Psi|^{2}}{30+|\Psi|^{2}}, \\
\Psi(0)+\Psi(1)=\frac{\cos (\Psi)}{10}, \quad 0.4 \Psi^{\prime}(0)+0.4 \Psi^{\prime}(1)=\frac{\sin (\Psi)}{20} .
\end{array}\right.
$$

As regards Theorem 3.4, it is not difficult to see that all the hypotheses are satisfied. Thus, the said problem has a solution in $\Upsilon$. For the uniqueness we presume $K_{\Psi}=\frac{1}{16}, K_{g_{1}}=\frac{1}{10}$, $K_{g_{2}}=\frac{1}{20}, K_{I}^{1}=\frac{1}{30}, K_{J}^{1}=\frac{1}{30}, l=1$, and by simple computation we have

$$
\begin{aligned}
K= & \left(\frac{1}{\frac{3}{4} \times \sqrt{(\pi)}}+\frac{0.5 \times 1}{\frac{3}{4} \times \sqrt{(\pi)}}+\frac{0.5}{\frac{3}{4} \times \sqrt{(\pi)}}+\frac{1.25}{\frac{1}{2} \times \sqrt{(\pi)}}+\frac{0.25}{\frac{1}{2} \times \sqrt{(\pi)}}\right) \times \frac{1}{16} \\
& +\left(0.5 \times \frac{1}{30}\right)+\left(0.25 \times \frac{1}{30}\right)+\left(1.875 \times \frac{1}{20}\right)+\left(0.5 \times \frac{1}{10}\right) \\
= & \frac{357}{1000} .
\end{aligned}
$$

From (4.1), we see $K<1$. Hence all the conditions of Theorem 3.5 hold. Consequently the given problem (1.1) under RBCs has a unique solution in $\Upsilon$.

\section{Conclusion}

With the support of a degree method of topology, we found sufficient conditions for the impulsive problem (1.1) and by a well-known Banach contraction theorem the uniqueness conditions have been obtained. A test example is presented and it is observed that our results are effective under some conditions. 
Funding

The authors received no direct funding for this work.

\section{Availability of data and materials}

Not applicable.

\section{Competing interests}

The authors declare that they have no competing interests.

Authors' contributions

The authors declare that they read and approved the final manuscript.

\section{Author details}

'Department of Mathematics, University of Malakand, Chakadara Dir(L), Pakistan. ${ }^{2}$ Department of Mathematics and General Sciences, Prince Sultan University, Riyadh, Saudi Arabia. ${ }^{3}$ Department of Medical Research, China Medical University, Taichung, Taiwan. ${ }^{4}$ Department of Computer Science and Information Engineering, Asia University, Taichung, Taiwan. ${ }^{5}$ College of Engineering and Technology, American University of the Middle East, Egaila, Kuwait.

\section{Publisher's Note}

Springer Nature remains neutral with regard to jurisdictional claims in published maps and institutional affiliations.

Received: 11 April 2020 Accepted: 8 May 2020 Published online: 15 May 2020

\section{References}

1. Hilfer, R:: Applications of Fractional Calculus in Physics. World Scientific, Singapore (2000)

2. Miller, K., Ross, B.: An Introduction to the Fractional Calculus and Fractional Differential Equations. Wiley, New York (1993)

3. Podlubny, I.: Fractional Differential Equations. Mathematics in Science and Engineering. Academic Press, New York (1999)

4. Tian, Y., Ge, W.: Applications of variational methods to boundary-value problem for impulsive differential equations. Proc. Edinb. Math. Soc. 51(2), 509-527 (2008)

5. Kilbas, A.A., Srivastava, H.M., Trujillo, J.J.: Theory and Applications of Fractional Differential Equations. North-Holland Mathematics Studies. Elsevier Amsterdam (2006)

6. Abdeljawad, T., Jarad, F., Baleanu, D.: On the existence and the uniqueness theorem for fractional differential equations with bounded delay within Caputo derivatives. Sci. China Math. 51(10), 1775-1786 (2008)

7. Momani, S., Zeidan, D.: Proceedings of International Conference on Fractional Differentiation and Its Applications (ICFDA) (2018)

8. Yavuz, M.: Characterizations of two different fractional operators without singular kernel. Math. Model. Nat. Phenom. 14(3), 302 (2019)

9. Yavuz, M., Özdemir, N.: Comparing the new fractional derivative operators involving exponential and Mittag-Leffler kernel. Discrete Contin. Dyn. Syst., Ser. S 13(3), 995 (2020)

10. Keten, A., Yavuz, M., Baleanu, D.: Nonlocal Cauchy problem via a fractional operator involving power kernel in Banach spaces. Fractal Fract. 3(2), 27 (2019)

11. Samoilenko, A.M., Perestyuk, N.A.: Impulsive Differential Equations. World Scientific, Singapore (1995)

12. Xu, D., Huang, Y., Liang, L.: Existence of positive periodic solution of an impulsive delay fishing model. Bull. Math. Anal. Appl. 3(2), 89-94 (2011)

13. Shah, K., Khalil, H., Khan, R.A.: Investigation of positive solution to a coupled system of impulsive boundary value problems for nonlinear fractional order differential equations. Chaos Solitons Fractals 77, 240-246 (2015)

14. Promsakon, C., Suntonsinsoungvon, E., Ntouyas, S.K., Tariboon, J.: Impulsive boundary value problems containing Caputo fractional derivative of a function with respect to another function. Adv. Differ. Equ. 2019(1), 486 (2019)

15. Ahmad, B., Wang, G.: A study of an impulsive four point boundary value problem of nonlinear fractional differential equations. Comput. Math. Appl. 62, 1341-1349 (2011)

16. Chen, A., Chen, Y.: Existence of solutions to anti-periodic boundary value problem for nonlinear fractional differential equations with impulses. Adv. Differ. Equ. 2011, Article ID 915689 (2011)

17. Simeonov, P.: Impulsive Differential Equations: Periodic Solutions and Applications. Routledge, London (2017)

18. Mawhin, J.: Topological Degree Methods in Nonlinear Boundary Value Problems. Am. Math. Soc., Providence (1979)

19. Isaia, F.: On a nonlinear integral equation without compactness. Acta Math. Univ. Comen. 2, 233-240 (2006)

20. Wang, J., Zhou, Y., Wei, W.: Study in fractional differential equations by means of topological degree methods. Numer. Funct. Anal. Optim. 33(2), 216-238 (2012)

21. Shah, K., Khan, R.A.: Existence and uniqueness results to a coupled system of fractional order boundary value problems by topological degree theory. Numer. Funct. Anal. Optim. 37(7), 887-899 (2016)

22. Ali, N., Shah, K., Baleanu, D., Arif, M., Khan, R.A.: Study of a class of arbitrary order differential equations by a coincidence degree method. Bound. Value Probl. 2017, 111 (2017)

23. Shah, K., Hussain, W.: Investigating a class of nonlinear fractional differential equations and its Hyers-Ulam stability by means of topological degree theory. Numer. Funct. Anal. Optim. 40(12), 1355-1372 (2019)

24. Anderson, M.L., Andrew, P.B., Fowkes, N.: Boundary tracing and boundary value problems: II. Applications. Proc. R. Soc. A, Math. Phys. Eng. Sci. 463(2084), 1925-1938 (2007)

25. Ahmad, B., et al.: Recent trends in boundary value problems. Abstr. Appl. Anal. 2013, Article ID 261754 (2013)

26. Mei, Z.: Numerical Bifurcation Analysis for Reaction-Diffusion Equations. Springer, Berlin (2000)

27. Hahn, D.W., Ozisk, M.N.: Heat Conduction, 3rd edn. Wiley, New York (2012) 